\title{
PERFORMANCE ANALYSIS OF DIGITAL BEAMFORMING ALGORITHMS
}

\author{
Sushma K M${ }^{1}$, Manjula Devi T $\mathbf{H}^{2}$ \\ ${ }^{1}$ Digital Communication and Networking and Telecommunication Department \\ ${ }^{2}$ Dayanand Sagar College of Engineering, Bangalore
}

\begin{abstract}
Interference reduction is needed for being able to effectively communicate with mobile users. One method that can be used as a solution to reduce interference is using smart antenna technology. Along with interference reduction there is increase in capacity and coverage. Adaptive beam forming techniques forms a narrow beam and directs it towards desired users and thereby forms nulls in interference directions This improves signal to noise ratio. The study documented in this paper shows the performance of variants of the Least Mean Square (LMS) Algorithm based approaches, in the realm of beam formation, used earlier in adaptive filtering, aiming at reduction in complexity, without considerable degradation in performance. The proposed algorithms namely Signum-Error Least Mean Square Algorithm along with LMS Algorithm are tested for computational complexity in weight vector updating and observed that the ones proposed perform better than the conventional Least Mean Square algorithm with respect to reduced complexity in beam formation, making them suitable for high speed digital communication systems. This paper will analyze Least Mean square algorithm and Sample Matrix Inverse algorithm for the performance. The consequence will demonstrate the Least Mean Square as a Solution to jammer cancellation when compared to Sample Matrix Inverse.
\end{abstract}

Keywords: Smart Antenna, Beamforming, Interference, Adaptive array, Uniform linear array. ****

\section{INTRODUCTION}

In recent years a substantial increase in the development of broadband wireless access technologies for evolving wireless internet services and improved cellular systems has been observed. It is widely foreseen that in the future an enormous rise in traffic for mobile and personal communication systems shall arise, due to an increase in number of users and introduction of new high bit rate data services. There are certain negative factors like co-channel interference and fading in the radiation environment contributing to the limit in the capacity. Smart antenna is a development in this direction to fulfill the feature requirements of mobile networks. A smart antenna is multiple antennas that perform spatial signal processing using different algorithms for direction of arrival estimation and for beamformation. As said, two basic functions of any smart antenna are: DOA Estimation and Adaptive Beam Forming (ABF). In digital beamforming, signals are converted from radio frequencies to an intermediate frequency, and converted to digital form and then down converted. Then this signal is given to beamforming algorithms that are burnt on digital signal processors. In this technology digital signal processing is used to estimate the direction in which incoming RF energy is incident on an array of antenna elements. Digital Beamforning receivers multiply each user's signal by complex weight vectors that adjust the excitation amplitudes and phases of the signal from each antenna element. In this paper, Least Mean Square (LMS) adaptive algorithm is chosen because of its simplicity. Jammer reduction can be implemented using an array with steered beams. If we use directional beams to communicate with mobiles on the downlink, interference between the base station and its nearby co-channel base stations will be less than if it used an Omni directional antenna. With Adaptive antennas a base station can communicate with two or more mobiles on the same frequency using SDMA, where, many mobiles communicate with a single base station on the same frequency. Using highly directional beams and/or forming nulls in the directions of all but one of the mobiles on a frequency, the base station creates multiple channels using the same frequency, but separated in space. Smart or adaptive antenna systems can improve link quality by combating the effects of multipath propagation or constructively exploiting the different paths, and increase capacity by reducing interference and allowing transmission of different data streams from different antennas as well. This paper will address an approach to reduce interference in a noisy environment as a means to enhance intelligible communications from one mobile user to another. Lastly, results presented will illustrate the utility of LMS algorithm in a congested environment as a means to null out potential jammers. 


\section{RELATED RESEARCH WORK}

The latest advancements in the field of smart antenna, understanding of Direction of Arrival and Beamforming algorithms are explicitly described in the following papers. Suzana Lamar [1] has explained about LMS algorithm. This algorithm does estimation of correlation between successive error samples. With this step size is controlled. George V Tsoulos [2] presents a general overview of smart antenna along with their application and potential benefits for mobile communication systems. Seungwon Choi [4] begins with the description of various types of smart antenna and then provides information about multiple benefits involved such as increased transmit power and capacity. It reaffirms the fact that utilizing different characteristics of smart antennas can lead to several operational benefits for a communication system. Jack $\mathrm{H}$. Winters [5] in his paper gives an overview about the need for Smart Antennas (SA) in mobile systems. The paper briefs about the various Beam forming and DOA algorithms along with their hardware implementation. Finally the author has concluded the fact that, the implementation of SA has little impact on the physical layer thereby improving the Quality of Service (QoS) and reducing interference at the same time.

\section{BEAMFORMER}

A Beamformer is a set of antennas arranged in a linear fashion with outputs that can be electronically steered. The sensor array consists of isotropic antenna elements that take spatial data from the waves emitted by signal sources. The signal received at these sensors is sent for computation of weights. Beamforming can be defined as adjusting the sensitivity of antenna in a particular direction for a given frequency. Here signals are isolated according to their directional characteristics and frequency content. Thus, a beam former can be viewed as a spatio-temporal filter. The aim of beamforming is to isolate the signal of the desired user from interference and noise. The weights of the filter are continuously changed and are adapted according to the received signal. Such adaptive filters attempt to filter out jammer signals, which are received by the antennas from directions other than the required signal source.

\section{SWITCHED BEAMFORMING}

In Switched Beamforming the array weights does not depend on the input or output array signals. The complex weights are selected to form a beam in particular, predetermined directions, from an existing library of weights. Switched beam antenna systems form multiple fixed beams with increased sensitivity in specific directions. These systems find out the signal strength, chosen from one of several predetermined, fixed beams and switch from one beam to another as the mobile user moves throughout the range. Instead of shaping the directional beam pattern with the metallic properties and physical design of a single element, switched beam systems combine the outputs of multiple antennas in such a way as to form directional beams with more spatial selectivity than can be gained with conventional, one-element approaches.

\section{DIGITAL BEAMFORMING}

Adaptive Beamforming is an advanced approach to beamforming. This is a technique in which an array of antennas is exploited to achieve maximum reception in a specified direction while rejecting signals of the same/different frequency from other directions. The weights are computed and adaptively updated in real time based on signal samples. Doing so, it's easy to track the user directions continuously. The adaptive process permits narrower beams in look direction and reduced output in other directions, which results in significant improvement in Signal to Interference Noise Ratio (SINR). With this technology, each user's signal is transmitted and received by the base station only in the direction of particular user. Thus there will be drastic reduction in overall interference in the system. This is improved by varying the weights applied to each of the antennas used in the array.

\section{SAMPLE MATRIX INVERSE}

SMI is used if the desired and jamming signals are known before or have been estimated. This provides the direct and fastest solution to compute the optimal weights. However, if the signals are not known exactly, then signal environment undergoes frequent changes. Thus, the signal processing unit must continuously update the weight vector to meet the new requirements imposed by the varying conditions. The weight vector must be updated without a priori information which, leads to estimation of covariance matrix $R_{x x}$ and cross-correlation vector $\boldsymbol{r}_{x s}$ in a finite observation interval given by equations

$$
\begin{aligned}
& R_{x x}=E\left[X X^{H}\right] \\
& r_{x s}=E\left[X S^{H}\right]
\end{aligned}
$$

Where, $X$ is induced signal matrix, $X^{H}$ is hermitian transpose of $X, E$ is the expectation operator and $S$ is reference signal matrix. The equation to compute Lx1 weight vector to steer beam in look direction and direct nulls at interfering source directions by using SMI algorithm is given by

$$
w(n)=R_{x x}^{-1} r_{x s}
$$

Where, $R_{x x}^{-1}$ is inverse of autocorrelation matrix $R_{x x}$ and $r_{x s}$ is cross-correlation. 


\subsection{Simulation Methodology of SMI}

1. Compute the Lx1 steering vector for desired direction $\theta_{0}$.

$$
a(\theta)=\left[\begin{array}{c}
1 \\
e^{i 2 \pi d \sin \theta} \\
\vdots \\
\vdots \\
e^{i 2 \pi d(L-1) \sin \theta}
\end{array}\right]
$$

2. Compute the LxM array manifold vector corresponding to $\mathrm{M}$ interference source directions $\theta_{1}, \theta_{2}, \ldots \ldots . ., \theta_{M}$

$$
A=\left[\begin{array}{cccc}
1 & 1 & \ldots \ldots & 1 \\
e^{i 2 \pi d \sin \theta_{1}} & e^{i 2 \pi d \sin \theta_{2}} & \ldots \ldots & e^{i 2 \pi d \sin \theta_{M-1}} \\
\vdots & \vdots & \vdots & \vdots \\
e^{i 2 \pi d(L-1) \sin \theta_{1}} & e^{i 2 \pi d(L-1) \sin \theta_{2}} & \ldots \ldots & e^{i 2 \pi l(L-1) \sin \theta_{(M-1)}}
\end{array}\right]
$$

3. Obtain signal samples ' $S$ ' by sampling continuous time signal of baseband frequency.(for simulation cosine wave samples are considered)

4. Compute Lx 1 cross-correlation matrix $r_{x s}$ by using

$$
r_{x s}=E\left\lfloor X S^{H}\right\rfloor
$$

5. Compute LxL covariance matrix $\boldsymbol{R}_{x x}$ by using

$$
R_{x x}=E\left[X X^{H}\right]
$$

6. The inverse of covariance matrix $\boldsymbol{R}_{x x}$ is found.

7. The weight vector is computed by using equation

$$
w(n)=R_{x x}^{-1} r_{x s}
$$

8. The array factor is computed by using equation

$$
A F=\sum_{i=1}^{L} w^{H}(i) e^{j 2 \pi d \sin (\theta)} \quad-90^{0} \leq \theta+0.001 \leq+90^{0}
$$

The value of $\theta$ in equation varies between $-90^{\circ} \leq \theta+0.001 \leq+90^{\circ}$ and $w^{H}(i)$ is hermitian transpose weight update vector $w(n)$.

9. Array factor versus angles are plotted.

\section{LEAST MEAN SQUARE}

Antenna arrays can be used in dynamic environments, where both the desired and interfering signals arrive from changing directions and with varying powers. Antenna arrays employ adaptive weighting algorithms that change the weights based on the received signals to increase the performance of the array. This weighting process aids in directing the antenna beam to point in the direction of the mobile user, and provides the capability for nulling out potential jammers. The LMS algorithm is one of the most widely used adaptive algorithms. The primary objective for the LMS algorithm is to adaptively produce weights that decrease the mean squared error between a desired signal and the arrays output. Thereby, increasing the reception in the direction of the desired signal and reduced reception from the interfering signal. The LMS algorithm is most commonly used because of its simplicity and reasonable performance. Since it is an iterative algorithm it can be used in a highly time varying signal environment. Also, there are several variants of the LMS algorithm that deal with the shortcomings of its basic form

\subsection{Simulation Methodology of LMS}

1. Compute the Lx1 steering vector for desired direction $\theta_{0}$.

$$
a(\theta)=\left[\begin{array}{c}
1 \\
e^{i 2 \pi d \sin \theta} \\
\vdots \\
\vdots \\
e^{i 2 \pi d(L-1) \sin \theta}
\end{array}\right]
$$

2. Compute the LxM array manifold vecto corresponding to $\mathrm{M}$ interference source directions $\theta_{1}, \theta_{2}, \ldots \ldots \ldots, \theta_{M}$.

$$
\text { ]. } A=\left[\begin{array}{cccc}
1 & 1 & \ldots \ldots & 1 \\
e^{i 2 \pi d \sin \theta_{1}} & e^{i 2 \pi l \sin \theta_{2}} & \ldots \ldots & e^{i 2 \pi l \sin \theta_{M-1}} \\
\vdots & \vdots & \vdots & \vdots \\
e^{i 2 \pi l(L-1) \sin \theta_{1}} & e^{i 2 \pi d(L-1) \sin \theta_{2}} & \ldots \ldots . & e^{i 2 \pi d(L-1) \sin \theta_{(M-1)}}
\end{array}\right]
$$

3. Obtain signal samples ' $S$ ' by sampling continuous time signal of baseband frequency. (For simulation sine wave samples are considered).

Compute the autocorrelation matrix $\boldsymbol{R}_{x x}$.

4. Compute the step size by using

$$
\mu=\frac{2}{\lambda_{\max }+\gamma}
$$


5. Compute the following for all signal samples $0 \leq n \leq N_{s}$.

Where, $N_{s}$ is the total number of signal samples

$$
\begin{gathered}
x(n)=a\left(\theta_{0}\right) s(n)+i(n) \sum_{i=1}^{M} a\left(\theta_{i}\right)+n_{0}(n) \\
y(n)=w(n)^{T} x(n) \\
e(n)=s(n)-y(n)
\end{gathered}
$$

6. The array factor is calculated as

$$
A F=\sum_{i=1}^{L} w^{H}(i) e^{j 2 \pi d \sin (\theta)}-90^{0} \leq \theta+0.001 \leq+90^{0}
$$

7. Array factor versus angles are plotted

\section{RESULTS}

Table 1: Inputs to the algorithms for less elements

\begin{tabular}{|l|l|l|l|}
\hline Algorithms & $\begin{array}{l}\text { Antenna } \\
\text { Elements }\end{array}$ & $\begin{array}{l}\text { Look } \\
\text { Direction }\end{array}$ & $\begin{array}{l}\text { Jammer } \\
\text { Directions }\end{array}$ \\
\hline SMI & 8 & 45 & {$[10,60,70]$} \\
\hline LMS & 8 & 45 & {$[10,60,70]$} \\
\hline
\end{tabular}

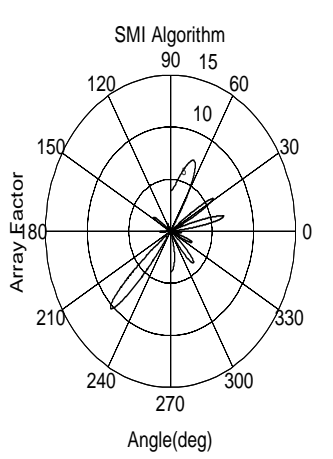

Fig- 1: Polar Plot of SMI for 8 antenna elements

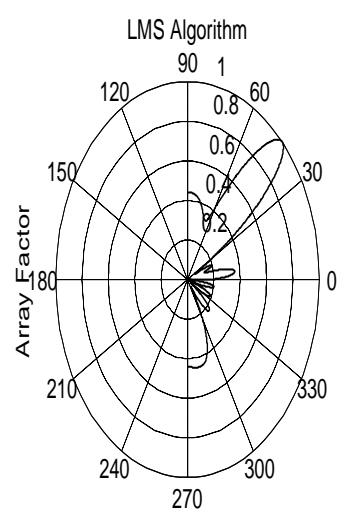

Angle(deg)

Fig- 2: Polar Plot of LMS for 8 antenna elements

Table 2: Inputs to the algorithms for more elements

\begin{tabular}{|l|l|l|l|}
\hline Algorithms & $\begin{array}{l}\text { Antenna } \\
\text { Elements }\end{array}$ & $\begin{array}{l}\text { Look } \\
\text { Direction }\end{array}$ & $\begin{array}{l}\text { Jammer } \\
\text { Directions }\end{array}$ \\
\hline SMI & 100 & 45 & {$[10,60,70]$} \\
\hline LMS & 100 & 45 & {$[10,60,70]$} \\
\hline
\end{tabular}

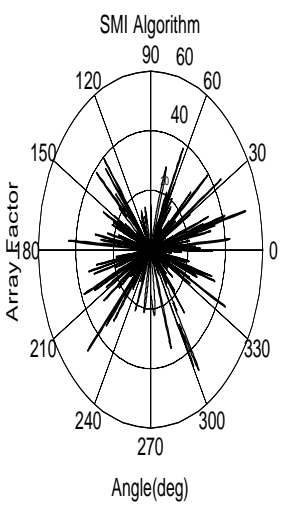

Fig- 3: Polar Plot of SMI for 100 antenna elements

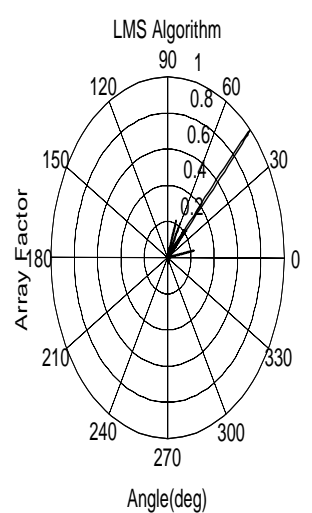

Fig- 4: Polar Plot of LMS for 100 antenna elements 


\section{DISCUSSION}

Here, we first compared the adaptive algorithm with the conventional approach for a uniform linear array of 8 and 100 elements. The spacing of every two array elements is half wavelength in order to avoid grating lobe. Digital beam forming (DBF) technology is progressed with the development of adaptive algorithms and architectures Modern beam forming systems Digital beamforming enables full utilization of the maximum number of degrees of freedom in the array.Least Mean Square (LMS) algorithm is being chosen to update complex weights to form the beam in the desired direction. Adaptive Beamforming is the process of recursively updating the complex weights for each of the antenna elements used in the array to achieve maximum reception in desired direction. The Adaptive DBF receiver performs a highly selective spatial filtering of arriving signals. Diverse adaptive beamforming algorithms with varying complexities have been widely used in different areas such as civilian mobile communications, sonar, radar, radio astronomy etc. Most adaptive beamforming algorithms are concerned with the maximization of the signal to noise ratio. We have chosen LMS as an adaptive algorithm because it is very simple and easy to apply since it requires no calculations or complex measures. This work can also be extended for other adaptive algorithms depending on the need and application. Use of adaptive antenna in existing systems not only reduces power consumption and interference but also increases spectral density in wireless communication. The contribution to a greener environment is twofold - while unwanted radiation exposure is reduced the consumption of diesel fuels that energize the base stations and their transmitters, are also reduced. Some recent survey based reports by well known environmental agencies in India [8] indicates very heavy diesel consumption by base stations. The use of smart antenna promises a drastic reduction of diesel consumption in cellular communications.

\section{CONCLUSIONS}

The simulation results for 8 and 100 ULA of antenna elements. The distance between adjacent antenna elements is lamda by 2 . The primarily used for this experiment was Matlab software. The antenna outputs will be pure sinusoidal wave with changed amplitudes and phases, and for each element in the array there is a time delay at which the signal arrives at each element. Both the SMI and LMS beamforming algorithms were discussed, and the LMS beam former demonstrated its ability to direct the antenna beam in the direction of the mobile user as well as its ability to null out the jammers. This technique is significant in highly time varying signal environments and could serve as a potential solution to jammer rejection. Figure $[3,4]$ clearly shows that SMI fails to form beam in the look direction without interference, where as LMS works well in beam formation and reduced jamming effect compared to SMI.

\section{REFERENCES}

[1] Suzanna LaMar1, Hugh Nguyen, and Paul Zavidniak, "Beamforming Solutions for Interference Reduction for High Altitude Airborne CDMA Systems". IEEE-2011.

[2] George V Tsoulo, "Smart antennas for mobile communication systems: Benefits and Challenges", IEEE proceedings of international conference on information technology, Vol-11, Issue-2 ,pp 84-94.

[3] D. G. Manolakis, Vinay K Ingle and Stephen M Kogon, "Statistical and Adaptive Signal processing spectral estimation, signal modeling, Adaptive filtering and array processing," Mc Graw Hill 2000.

[4] Seungwon Choi, Hong-Min Son, and Tapan K sarkar, "Implementation of a Smart Antenna System on a General-Purpose Digital Signal Processor Utilizing a Linearized CGM", Digital Signal Processing Journal, July-1997, Vol-7, pp 105-119.

[5] Jack H. Winters, "Smart Antennas for Wireless Systems" , proceedings of IEEE international conference on signal processing, June 2005, pp 107-109

[6] Sungsoo, Seungwon and Tapan K sarkar, "An Adaptive Beamforming Algorithm with a Linear Complexity for a Multipath Fading CDMA Channel", IEICE Transaction.

[7] "Diesel to Power Telecom Towers - The Big Problem", authored by VNL Pvt Ltd India, available at the URL cited:

http://www.vnl.in/blog/2012/diesel-to-power-telecom-to wers-the-big-problem/ published on 5 Apr, 2012.

\section{BIOGRAPHIES}

Sushma K.M received B.E in Electronics and Communication from PDIT, Hospet. Pursuing M.Tech in Digital Communication and Networking from DSCE, Banglore.

Manjula Devi T.H currently working as Associate Professor in Dayanand Sagar College of Engineering, Bangalore. She has received B.E in Electronics and Communication and M.E in Electronics and Communication and pursuing Ph.d in Image Processing. Her area of interest includes Image Prosessing, DSP and Communication. 